\title{
AVERAGE GAUSSIAN CURVATURE OF LEAVES OF FOLIATIONS
}

\author{
BY DANIEL ASIMOV 1 \\ Communicated by P. T. Church, July 26, 1977
}

Let $F$ be a smooth transversely-oriented foliation of a compact, connected, oriented, Riemannian manifold $W^{n+1}$ of constant sectional curvature $\equiv c$. Let $K_{\mathrm{F}}: W \rightarrow \mathbf{R}$ via $K_{\mathrm{F}}(x)=$ the Gaussian curvature (defined below) of the leaf $l^{n}$ through $x$ at $x$. For $n=2$ this is classical Gaussian curvature. Let vol be the canonical volume on $W$, and define $\bar{K}_{\mathrm{F}}$ by Volume $(W) \cdot \bar{K}_{\mathrm{F}}=\int_{W} K_{\mathrm{F}}$ vol.

THEOREM 1.

$$
\bar{K}_{F}= \begin{cases}2^{n} c^{n / 2} /\left(\begin{array}{c}
n \\
n / 2
\end{array}\right), & n \text { even, } \\
0, & n \text { odd } .\end{cases}
$$

TheOREM 2. Let $n+1=3$ and suppose $F, W, c$ are as above except that $\partial W$ is nonempty and is a union of leaves of $F$. Then

$$
\int_{W} K_{\mathrm{F}} \mathrm{vol}=2 c \text { Volume }(W)+\int_{\partial W} H \text { vol }^{\prime}
$$

where $H: \partial W \rightarrow \mathbf{R}$ is the mean curvature (computed with respect to the transverse orientation), and $\mathrm{vol}^{\prime}$ is the canonical volume on $\partial W$.

Theorem 3. Suppose $n+1=3$. Let $F$ and $W$ be as in the original hypotheses with $\partial W=\varnothing$ but assume the sectional curvatures of $W$ lie between $c_{1}$ and $c_{2}$. Then we have $2 c_{1} \leqslant \bar{K}_{F} \leqslant 2 c_{2}$.

Definition of Gaussian Curvature. We define, for a Riemannian manifold $l=l^{n}$, the function $K: l \rightarrow \mathrm{R}$ in two cases (which overlap):

Case (i). $n$ is even. In this case a local orthonormal frame on $l$ gives rise to a matrix of curvature 2-forms, $\Omega=\left(\Omega_{j}^{i}\right)$ defined locally. The Pfaffians of the local $\Omega$ agree on overlaps and so define a global $n$-form $\operatorname{Pf}(\Omega)$ on $l$. Letting $\nu$ denote the canonical volume form on $l$ we set

(see [3, vol. V, pp. 417-420]).

$$
K \nu=\frac{2^{n / 2} \cdot(n / 2) !}{n !} \operatorname{Pf}(\Omega)
$$

AMS (MOS) subject classifications (1970). Primary 57D30, 53 C99.

Key words and phrases. Codimension-one foliation, Gaussian curvature, constant curvature.

1 Research supported by the National Science Foundation. 
Case (ii). Assume $l$ is a hypersurface of a flat Riemannian manifold $W$, and that $l$ is transversely oriented by a field of unit normals $\xi$. Then at each point $x$ of $l$ let $A_{x}: T_{x} l \rightarrow T_{x} l$ be defined by $A_{x} v=-\nabla_{v} \xi$. Then we define $K(x)=$ $\operatorname{det}\left(A_{x}\right)$. (See [3, vol. IV, p. 96].)

REMARKS. In the overlap of Cases (i) and (ii), viz. when $l$ is an even-dimensional hypersurface of a flat manifold, the two definitions of $K$ agree. If $n$ is even then $K$ is intrinsic to the geometry of $l$; if $n \geqslant 3$ is odd then $K$ is intrinsic up to a global choice of sign [3, vol. IV, p. 96].

Sketch of Proof of Theorem 1. We consider two cases: $n$ odd and $n$ even.

(i) The case $n$ is odd:

Here $\chi(W)=0$ and hence by Chern-Gauss-Bonnet [3, vol. V, p. 442] the constant curvature $c=0$, i.e. $W$ is flat. Without loss of generality we may assume, by taking a finite covering, that $W$ is in fact a flat torus $[1$, p. 212].

Let $T_{p} \approx \mathbf{R}^{n+1}$ denote the tangent space to $W$ at some point $p \in W . \mathbf{A}$ choice of unit normal vector field $\xi$ to the foliation $F$ determines (by parallel translation in $W$ ) a Gauss map $g: W \rightarrow T_{p}$ whose image lies of course in the unit sphere $S^{n} \subset T_{p}$. Think of $d g$ as a map $d g: W \rightarrow \operatorname{End}(T W)$ via $x \mapsto d g_{x}$.

Let $\sigma_{i}\left(E_{x}\right)$ denote the $i$ th elementary symmetric function of the eigenvalues of $E_{x}$, where $E_{x}$ is any endomorphism $E_{x}: T_{x} \rightarrow T_{x}$.

LEMMA. $K_{\mathrm{F}}(x)=\sigma_{n}\left(-d g_{x}\right)$, for all $x \in W$.

The proof is not difficult.

Now for each $t \in \mathbf{R}$ consider $h_{t}: W \rightarrow W$ defined by $h_{t}(x)=\exp (\operatorname{tg}(x))$, or in other words $h_{t}(x)=x+\operatorname{tg}(x)$ (by slight abuse of notation). A computation shows that

$$
\int_{W} J h_{t} \text { vol }=\int_{W} \operatorname{det}(I+t d g) \text { vol or }
$$

$$
\operatorname{Volume}(W)=\operatorname{Volume}(W) \cdot\left[1+\bar{\sigma}_{1}(d g) t+\cdots+\bar{\sigma}_{n}(d g) t^{n}\right]
$$

where $\bar{\sigma}_{i}(d g)$ denotes the average over $x \in W$ of $\sigma_{i}\left(d g_{x}\right)$, and $J$ denotes the Jacobian.

Since both sides of $(*)$ are polynomials in $t$ it follows that $\bar{\sigma}_{i}(d g)=0, i=$ $1, \ldots, n$.

COROllary. In the above case we have $\bar{\sigma}_{i}(d g)=0$ for $i=1, \ldots, n$. In particular $\sigma_{2}(d g)$ is a multiple of the leaf scalar curvature; hence the average leaf scalar curvature is 0 whenever $W$ is flat.

Sketch of Proof of Theorem 1 (Continued).

(ii) The case $n$ is even:

The proof depends on the construction of certain globally defined $n$-forms. Let $\left\{\theta^{1}, \ldots, \theta^{n}, \theta^{n+1}\right\}$ be a local adapted orthonormal coframe field (with 
$\theta^{n+1}$ orthogonal to the leaves of $F$ ) and let $\left\{\omega_{j}^{i}\right\}$ be the associated Riemannian connection forms. Put

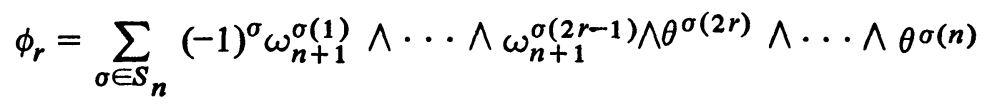

for $1 \leqslant r \leqslant n / 2$, where $S_{n}$ denotes the symmetric group on $\{1, \ldots, n\}$ and $(-1)^{\sigma}$ is the sign of the permutation $\sigma$.

Lemma. The $n$-forms $\phi_{r}$ do not depend on the choice of orthonormal coframe $\left\{\theta^{i}\right\}$ and hence are globally defined on $W$.

The proof is an unpleasant calculation.

Lemma. For each $n$ there exist constants $b_{r}, 1 \leqslant r \leqslant n / 2$ such that if we set

$(* *)$

$$
\Phi=\sum_{r=1}^{n / 2} b_{r} \phi_{r} \quad \text { then }
$$

$$
d \Phi=\left(K_{\mathrm{F}}-a_{n} c^{n / 2}\right) \text { vol where } a_{n}=2^{n} /\left(\begin{array}{c}
n \\
n / 2
\end{array}\right) .
$$

The proof is an even more unpleasant calculation.

Integrating (**) over $W$ readily yields $\bar{K}_{F}=2^{n} c^{n / 2} /\left(\begin{array}{c}n \\ n / 2\end{array}\right)$ as desired.

Remarks. By taking double covers we may prove Theorem 1 even if $W$ is allowed to be nonorientable. If $n$ is even then we may similarly drop the assumption that $F$ is transversely orientable. If $n$ is odd, however, transverse orientability is required in order that $K_{F}$ be defined.

Theorem 1 has been generalized in various ways in the recent paper of Rosenberg, Brito and Langevin [2]. Theorems 2 and 3 are proved using methods similar to Theorem 1.

\section{REFERENCES}

1. S. Kobayashi and K. Nomizu, Foundations of differential geometry. I, Interscience, New York and London, 1963.

2. F. Brito, R. Langevin and H. Rosenberg, Curvature integrals in foliated manifolds, Université Paris-Sud, Orsay, 1977 (preprint).

3. M. Spivak, A comprehensive introduction to differential geometry. IV, V, Publish or Perish, Boston, Mass., 1975.

INSTITUTE FOR ADVANCED STUDY, SCHOOL OF MATHEMATICS, PRINCETON, NEW JERSEY 08540

DEPARTMENT OF MATHEMATICS, UNIVERSITY OF MINNESOTA, MINNEAPOLIS, MINNESOTA 55455

DEPARTMENT OF MATHEMATICS, UNIVERSITY OF NORTH CAROLINA AT CHAPEL HILL, CHAPEL HILL, NORTH CAROLINA 27514

Current address: Department of Mathematics, Haverford College, Haverford, Pennsylvania 19041 